\title{
Amelanotic Primary Malignant Melanoma of the Maxilla: A Case Report
}

\author{
Mostafa Esmaeili, ${ }^{1}$ and Azam Hosseini ${ }^{1, *}$ \\ ${ }^{1}$ Tehran, IR Iran \\ "Corresponding author: Azam Hosseini, Tehran, IR Iran. Tel: +98-9123969130, E-mail: hosseini.a57@yahoo.com
}

Received 2017 January 08; Revised 2017 February 19; Accepted 2017 February 19.

\begin{abstract}
Introduction: Primary malignant melanoma of the oral cavity is a rare occurrence. This tumor, compared to other malignant tumors of the oral cavity, has higher tendency to metastasize or locally invade the adjacent structures. Its most common site of occurrence in the oral cavity is the palate followed by the maxillary gingiva. Oral melanoma does not have a pathognomonic clinical manifestation and in rare cases, it may not even cause clinical discoloration of the mucosa either; in this case, it is referred to as amelanotic melanoma. Most cases of amelanotic melanomas are metastatic rather than primary tumors. The Survival rate of patients with mucosal melanoma is lower than that of patients with cutaneous melanoma. Prognosis is much poorer for amelanotic melanoma due to difficult and delayed diagnosis compared to cutaneous melanoma.

Case Presentation: In this study, a 33-year-old white male presented to the oral medicine department of Shahed University, school of dentistry, complaining of an extensive pink-redexophytic mass on the attached gingiva of the maxillary right central incisor extending posteriorly to the maxillary right first molar. He had it for the past five months. The patient had no systemic disease and the panoramic radiograph of the patient showed no pathological lesion.
\end{abstract}

Keywords: Oral Primary Malignant Melanoma, Amelanotic Melanoma

\section{Introduction}

Malignant melanoma is a potentially progressive tumor with melanocytic origin (1). Primary mucosal melanoma accounts for less than $1 \%$ of all melanomas $(2,3)$. Palate followed by maxillary gingiva is the most common site of occurrence of oral melanoma (4). The prevalence of oral melanoma is higher among black people and Japanese compared to other populations $(5,6)$. Also, oral melanoma is more commonly seen in males compared to females and in patients over 50 years $(2,6)$.

Oral melanoma does not have a pathognomonic clinical manifestation. The most usual appearance is a brownblack macule at axillary gingiva or palate, which shows the radial growth phase on histopathologic examination. Later, with the onset of the vertical growth phase it may become nodular at clinical presentation $(1,7)$. In rare cases, melanoma may not cause clinical discoloration, which is called amelanotic melanoma (8). Most amelanotic melanomas are metastatic rather than primary tumors (911).

Prognosis of the mucosal melanoma is very poor. Amelanotic melanomas have a much poorer prognosis due to difficult and delayed diagnosis compared to melanotic melanomas (12). Herein, we report a case of amelanotic melanoma of the maxilla.

\section{Case Presentation}

A 33-year-old white male presented to the oral medicine department of Shahed University, School of Dentistry, complaining of a mass on his maxillary right gingiva. The patient reported that the lesion had been developed within the past five months in the form of a small mass between his maxillary right canine and the first premolar teeth and then rapidly grew. Within the first three months following the emergence of the lesion, the entire mass had been completely excised twice by a general dentist; however, unfortunately, the resected tissue had not been sent for histopathological examination.

Intraoral examination revealed an extensive pedunculated exophytic mass on the maxillary right attached gingiva extending from the central incisor posteriorly to the first molar tooth and covering the entire labial and buccal vestibule as well as the labial and buccal surfaces of the respective teeth. The mass had a pink-red color and firm consistency with a lobulated surface. Its surface was ulcerative and erosive in some areas (Figure 1). The lesion highly simulated reactive lesions such as pyogenic granuloma, peripheral giant cell granuloma and mesenchymal tumors. The patient had no pain in the area and had only presented for clinical examination of the mass. The patient was systemically healthy and was not on any medication. Panoramic and periapical radiographs did not show 
any pathological lesion (Figure 2). An incisional biopsy of the lesion was performed for histopathological examination, which revealed a neoplastic tumor with large sheets of round atypical cells with basophilic nuclei and various degrees of nuclear pleomorphism and hyperchromatism and numerous atypical mitoses. Moreover, invasive spindle-shaped or epithelioid cells in the form of ropes or sheets were noted. Brown melanin granules were evident in most areas and cytoplasm of the cells. Also, tumoral cells were extended to the margins of the biopsy specimen. Since the pathology report indicated melanoma, immunohistochemistry was performed for more accurate assessment, which confirmed the diagnosis of melanoma. According to the pathology report, magnetic resonance imaging (MRI) was requested to assess the extension of the lesion, which showed a bulky, lobulated mass. The lesion was isointenseto hyperintense on T1-weighted images and isointenseto hypointense on T2-weighted images. The magnetic resonance imaging with contrast revealed a nonhomogenous enhanced mass, which was slightly enhanced. The lesion was mainly in the lateral quadrant of the right maxillary ridge with no significant invasion to the adjacent tissues (Figures 3 and 4 ). The patient was referred to the Oral and Maxillofacial Surgery Department of Shahed University, School of Dentistry, for surgical resection of the lesion with a wide surgical margin. After surgical resection, surgical margin was confirmed to be free of tumor by pathological analysis and the pathology report for the entire resected mass was melanoma. Six months after hemi-maxillectomy, the patient presented again complaining of swelling and pain in the same region. So, MRI of the surgical site was requested. The MRI findings showed recurrence of the tumor in the previous area. Also, a wholebody positron emission tomography scan was requested for detection of any metastatic lesion and hopefully no metastatic lesion was detected. The patient underwent surgical resection again and scheduled for chemotherapy and radiotherapy. The combined regimens of hyperfractionated radiotherapy and chemotherapy were used. The patient followed 2 weeks, three and six months after the surgery. He was recovered after one month clinically and no signs or symptoms of recurrence were seen six months later clinically and also the new MRI was requested, which reported no sign of recurrence.

\section{Discussion}

Oral mucosalmelanoma is a rare malignancy (13). Despite its rarity, melanoma is the most important pigmented lesion of the oral cavity due to its poor prognosis and a high mortality rate. Thus, every single and stable pigmented lesion in the oral cavity must undergo biopsy

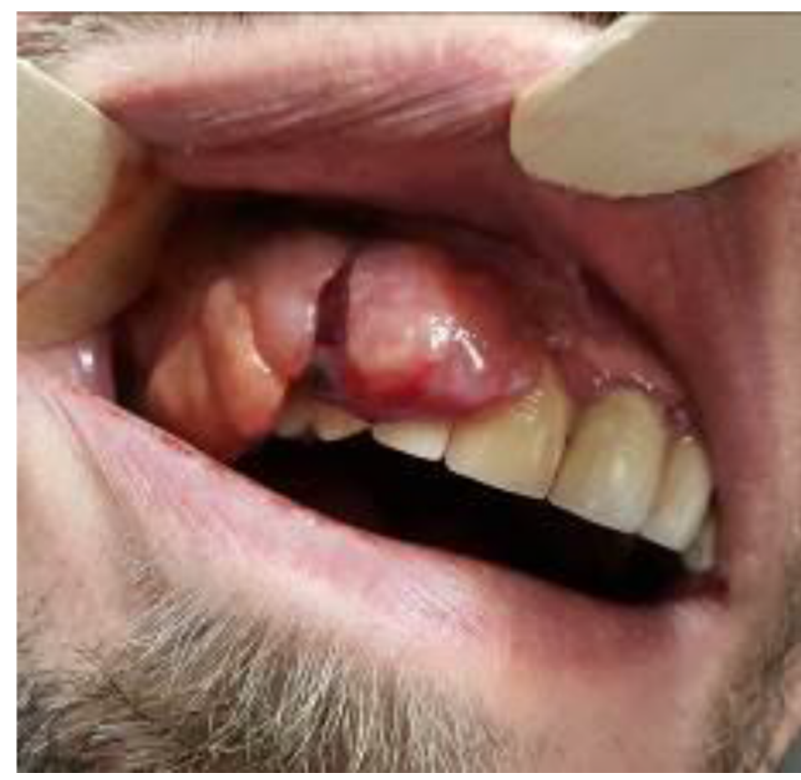

Figure 1. An Exophytic Mass in the Right Side of the Maxilla

to rule out malignant melanoma (9). Also, early diagnosis and in-time treatment of melanoma can decrease its related morbidity and mortality rates. Therefore, dentists play a pivotal role in early detection and diagnosis of melanoma (9). Oral melanoma often manifests in the form of a pigmented lesion, which can be black, brown, gray, purple or red or may even be devoid of pigmentation (amelanotic melanoma) (14). Also, it may have a smooth (macule) or prominent (nodule or tumor) surface clinically and can be ulcerative or nonulcerative. It may or may not have erythematous borders (9).

Dentists should be suspected of malignancy when they encounter a pigmented lesion with characteristics such as asymmetry, irregular borders, variation in color (red to black-brown), diameter of more than $6 \mathrm{~mm}$ and prominence of the mass (9). Other signs and symptoms of oral melanoma are nonspecific and resemble those of other malignancies; these signs and symptoms include ulceration, bleeding, pain, tooth mobility and delayed healing of extraction sockets (14). Regional lymphadenopathy may also be present, and indicates poor prognosis (15).

Our patient had a clearly visible mass in his mouth in the form of an exophytic lesion with areas of ulceration on his maxillary gingiva, highly resembling common reactive lesions of the gingiva such as pyogenic granuloma and peripheral giant cell granuloma. The lesion did not have the usual appearance of an oral melanoma described in text books and it was the histopathological analysis, which revealed its nature. This highlights the significance of per- 

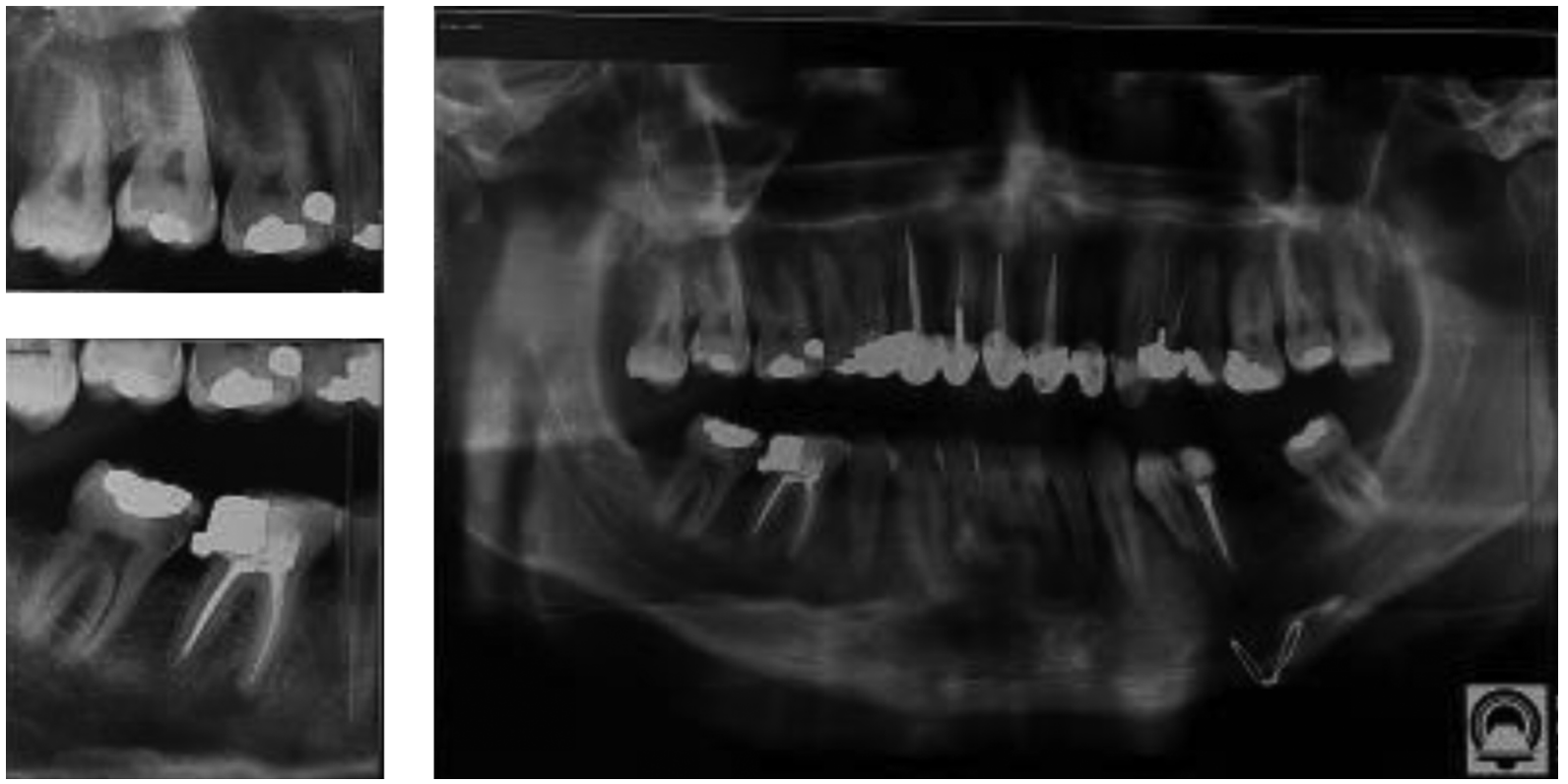

Figure 2. Panoramic and Periapical Views

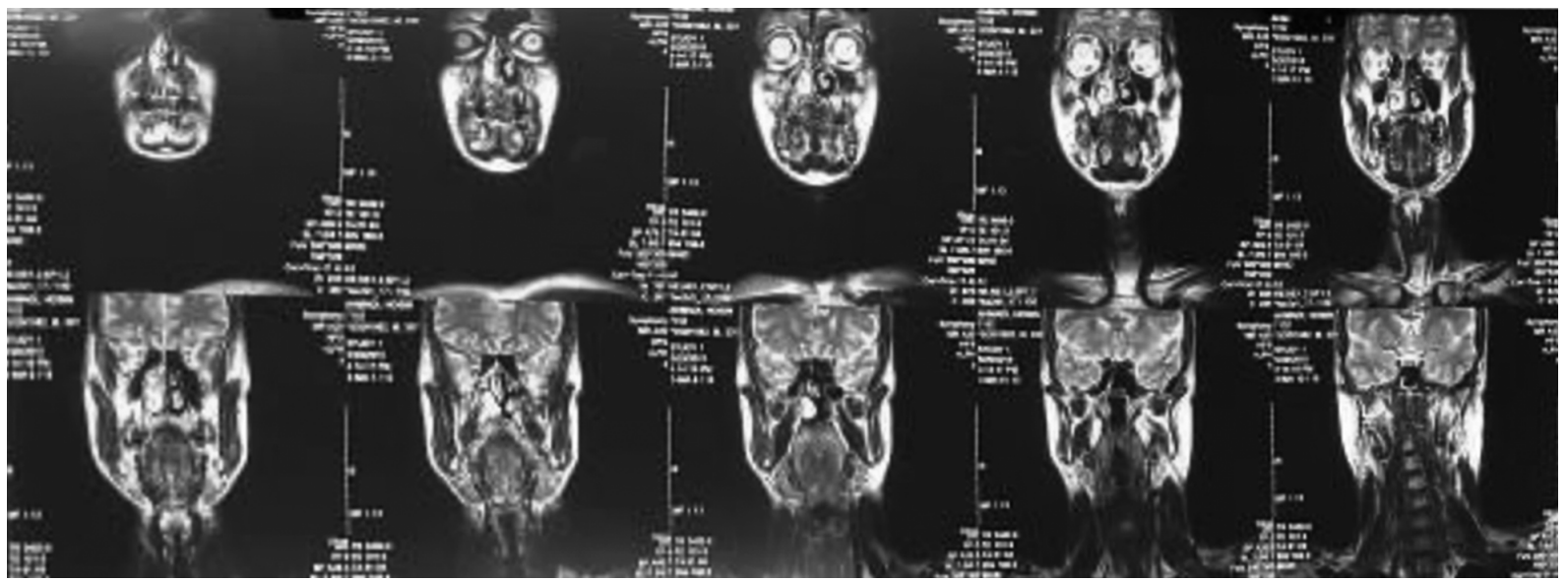

Figure 3. Frontal Sections of Magnetic Resonance Imaging

forming biopsy for all prominent lesions of the oral mucosa. High resemblance of this lesion to reactive lesions was due to its amelanotic type, which is extremely rare.

Melanoma has two growth phases:

1) Radial growth phase, which is the dominant phase during primary extension of melanoma in all types except for the nodular type. In this phase, malignant melanocytes extend along the basal layer horizontally.

2) Vertical growth phase: Malignant cells start to invade the underlying connective tissue. The vertical growth phase is dominant in the nodular type of melanoma with a very short or no radial phase at all (16).

Malignant melanoma has four clinicopathological types:

1) Superficial spreading melanoma: It is the most common form of melanoma accounting for $70 \%$ of cutaneous melanomas. It appears as a macule with different colors with the largest diameter of $3 \mathrm{~cm}$ reported at the time of diagnosis.

2) Nodular melanoma: It accounts for $15 \%$ of all cutaneous melanomas and appears in the form of a nodular prominence, which quickly invades the connective tis- 


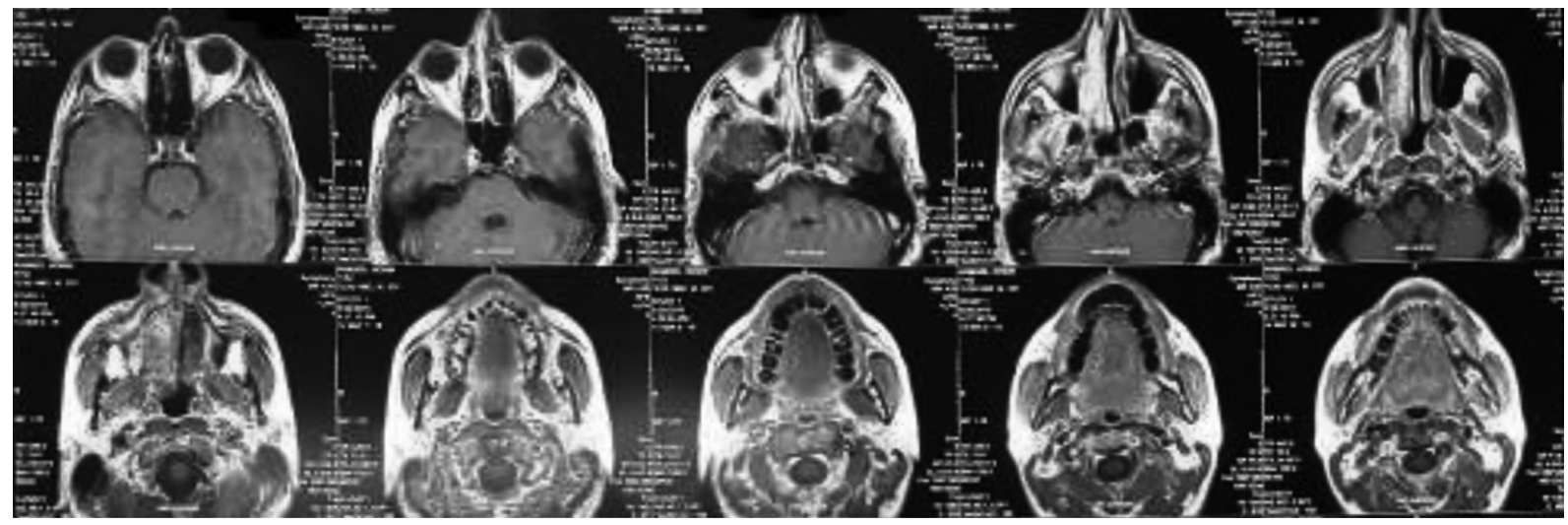

Figure 4. Axial Sections of Magnetic Resonance Imaging

sue. Nodular melanoma mainly appears as a severely pigmented exophytic lesion. However, in some cases, it is so poorly differentiated that creates a nonpigmented amelanotic melanoma.

3) Lentigomaligna melanoma: It accounts for 5\% - 10\% of the cutaneous melanomas and develops from a primary lesion called lentigomaligna (Hutchinson's freckle). Lentigomaligna occurs on the skin in areas exposed to sunlight particularly in the mid-face of white individuals. It transforms into an in-situ melanoma in the radial growth phase and appears as a large macule with slow expansion and irregular borders. Nodular appearance of lentigomaligna indicates initiation of the vertical or invasive growth phase and transformation to melanoma.

4) Acrallentiginous melanoma: It is the most common type of oral melanoma and the most common type of melanoma in black people occurring on the palms of the hands, the soles of the feet, in the nail bed and on mucous membranes. This type is more common in males (16).

Treatment of malignant melanoma depends on the extension of lesion and may include surgery, radiotherapy, chemotherapy and immunotherapy (6). In some cases with primary small lesions, surgical removal of the mass along with a minimum of $1.5 \mathrm{~cm}$ of healthy margin may suffice (16). Fine needle aspiration and exfoliative cytology are relatively contraindicated due to low diagnostic sensitivity (4).

Oral melanoma is highly invasive and abundance of blood supply in the oral mucosa often results in invasion of melanoma to the vasculature and its dissemination in primary stages of the disease (4). Also, according to the existing literature, the possibility of accidental seeding of malignant cells in the adjacent tissues, blood or lymph also exists during incisional biopsy of a malignant neoplasm; thus, an incisional biopsy of these lesions increases the risk of local recurrence and regional or distant metastasis. Radical dissection may be performed depending on the extension of tumor and level of involvement of lymph nodes (8).

Because detection of pigmented lesions in the oral cavity is more difficult than those on the skin, prognosis of oral melanoma is worse than that of cutaneous melanoma and is actually very poor with a 5-year survival rate of $10 \%$ - 25\%; this rate also depends on the site of lesion to some extent. For instance, the survival rate of patients with gingival melanoma is five years longer than that of patients with palatal melanoma $(5,9)$. Recurrence may occur 10 to 15 years after treatment of the primary tumor. Distant metastasis to the lungs, brain, liver and bone may occur in advanced stages of the disease (6).

Unfortunately, in our patient, the lesion had been excised twice by a general dentist with the assumption of being a simple reactive lesion such as the pyogenic granuloma but had not been sent for pathological analysis, which delayed the diagnosis. Considering the fact that amelanotic melanoma may mimic reactive lesions as well as the very poor prognosis of melanoma and the significance of its early detection, it is necessary to take a biopsy of all exophytic lesions for a thorough histopathological examination to rule out amelanotic melanoma and other malignancies such as squamous cell carcinoma and malignant mesenchymal tumors (17).

\subsection{Conclusions}

Primary oral malignant melanomas are very rare and have a high potential for malignancy. Oral melanomas may have a similar clinical manifestation as that of other pigmented oral lesions. All pigmented oral lesions, without definite clinical diagnosis, must undergo biopsy. Amelanotic melanomas may have high clinical resemblance 
to reactive lesions such as pyogenic granuloma and peripheral giant cell granuloma. Thus, even if reactive lesions are highly suspected, biopsy must be performed to histopathologically confirm the diagnosis because early diagnosis of melanoma can significantly improve the prognosis.

\section{References}

1. Sharma N. Primary oral malignant melanoma: Two case reports and review of literature. Case reports in dentistry. 2012;2012

2. Meleti M, Leemans CR, Mooi WJ, Vescovi P, van der Waal I. Oral malignant melanoma: a review of the literature. Oral Oncol. 2007;43(2):11621. doi: 10.1016/j.oraloncology.2006.04.001. [PubMed: 16931116].

3. Saghravanian N, Pazouki M, Zamanzadeh M. Oral amelanotic melanoma of the maxilla. J Dent (Tehran). 2014;11(6):721-5. [PubMed: 25628704].

4. Lopez-Graniel CM, Ochoa-Carrillo FJ, Meneses-Garcia A. Malignant melanoma of the oral cavity: diagnosis and treatment experience in a Mexican population. Oral Oncol. 1999;35(4):425-30. [PubMed: 10645410].

5. Hicks MJ, Flaitz CM. Oral mucosal melanoma: epidemiology and pathobiology. Oral Oncol. 2000;36(2):152-69. [PubMed: 10745167].

6. Gu GM, Epstein JB, Morton TJ. Intraoral melanoma: long-term follow-up and implication for dental clinicians. A case report and literature review. Oral Surg Oral Med Oral Pathol Oral Radiol Endod. 2003;96(4):404-13. doi: 10.1016/S1079210403003202. [PubMed: 14561964].

7. Adisa AO, Olawole WO, Sigbeku OF. Oral amelanotic melanoma. Ann Ib Postgrad Med. 2012;10(1):6-8. [PubMed: 25161399].
8. Tanaka N, Mimura M, Kimijima Y, Amagasa T. Clinical investigation of amelanotic malignant melanoma in the oral region. J Oral Maxillofac Surg. 2004;62(8):933-7. [PubMed: 15278856].

9. Rapini RP, Golitz LE, Greer RO Jr, Krekorian EA, Poulson T. Primary malignant melanoma of the oral cavity. A review of 177 cases. Cancer. 1985;55(7):1543-51. [PubMed: 3978548].

10. Vierne C, Hardy H, Guichard B, Barat M, Peron JM, Trost O. [Mandibular metastasis of a cutaneous melanoma or metachronous amelanotic melanoma of the oral cavity? A case report and literature review]. Ann Chir Plast Esthet. 2014;59(4):276-9. doi: 10.1016/j.anplas.2014.01.002. [PubMed: 24503521].

11. Matsuoka K. Oral malignant melanoma detected after resection of amelanotic pulmonary metastasis. Int J Surg Case Rep. 2013;4(12):116972. doi: 10.1016/j.ijscr.2013.10.004. [PubMed: 24189568].

12. Colllins B, LeonBarnes Jr E, Abernethy J. Oral malignant melanoma.J Clin Oncol. 2005;55:74-108.

13. Berthelsen A, Andersen AP, Jensen TS, Hansen HS. Melanomas of the mucosa in the oral cavity and the upper respiratory passages. Cancer. 1984;54(5):907-12. [PubMed: 6744219].

14. Barker BF, Carpenter WM, Daniels TE, Kahn MA, Leider AS, LozadaNur F, et al. Oral mucosal melanomas: the WESTOP Banff workshop proceedings. Western Society of Teachers of Oral Pathology. Oral Surg Oral Med Oral Pathol Oral Radiol Endod. 1997;83(6):672-9. [PubMed: 9195622].

15. Van der Waal I, Van der Kwast WAM. Malignant Melanoma. Oral Pathology, Quintessence. ;1988.

16. Damm DD, Bouquot JE, Neville BW, Allen C. Oral and maxillofacial pathology. Philadelphia: Saunders; 2002.

17. Pandiar D, Basheer S, Shameena PM, Sudha S, Dhana LJ. Amelanotic melanoma masquerading as a granular cell lesion. Case Rep Dent. 2013;2013:924573. doi: 10.1155/2013/924573. [PubMed: 23533832]. 